\title{
Hirzebruch $L$-polynomials and multiple zeta values
}

\author{
Alexander Berglund ${ }^{1}(D) \cdot J^{\prime}$.onas Bergström ${ }^{1}$
}

\begin{abstract}
We express the coefficients of the Hirzebruch $L$-polynomials in terms of certain alternating multiple zeta values. In particular, we show that every monomial in the Pontryagin classes appears with a non-zero coefficient, with the expected sign. Similar results hold for the polynomials associated to the $\hat{A}$-genus.
\end{abstract}

Mathematics Subject Classification Primary 55R40 - 11M32; Secondary 57R20

\section{Introduction}

The Hirzebruch $L$-polynomials are certain polynomials with rational coefficients,

$$
\begin{aligned}
& \mathrm{L}_{1}=\frac{1}{3} \mathrm{p}_{1} \\
& \mathrm{~L}_{2}=\frac{1}{45}\left(7 \mathrm{p}_{2}-\mathrm{p}_{1}^{2}\right) \\
& \mathrm{L}_{3}=\frac{1}{945}\left(62 \mathrm{p}_{3}-13 \mathrm{p}_{2} \mathrm{p}_{1}+2 \mathrm{p}_{1}^{3}\right)
\end{aligned}
$$

\section{Communicated by Thomas Schick.}

$\varangle \quad$ Alexander Berglund alexb@math.su.se

Jonas Bergström jonasb@math.su.se

1 Department of Mathematics, Stockholm University, 10691 Stockholm, Sweden 
featured in the Hirzebruch signature theorem, which expresses the signature $\sigma(M)$ of a smooth compact oriented manifold $M^{4 k}$ as

$$
\sigma(M)=\left\langle\mathrm{L}_{k},[M]\right\rangle,
$$

where $\mathrm{p}_{i}$ are taken to be the Pontryagin classes of the tangent bundle of $M$, see [3, Theorem 8.2.2] or [5, Theorem 19.4]. The $k$ th polynomial has the form

$$
\mathrm{L}_{k}=\mathrm{L}_{k}\left(\mathrm{p}_{1}, \ldots, \mathrm{p}_{k}\right)=\sum h_{j_{1}, \ldots, j_{r}} \mathrm{p}_{j_{1}} \cdots \mathrm{p}_{j_{r}}
$$

where the sum is over all partitions $\left(j_{1}, \ldots, j_{r}\right)$ of $k$, i.e., sequences of integers $j_{1} \geq$ $\cdots \geq j_{r} \geq 1$ such that $j_{1}+\cdots+j_{r}=k$. The purpose of this note is to establish certain properties of the coefficients $h_{j_{1}, \ldots, j_{r}}$.

For real numbers $s_{1}, \ldots, s_{r}>1$, we define the series

$$
T\left(s_{1}, \ldots, s_{r}\right)=\sum_{n_{1} \geq 2 \cdots \geq 2 n_{r} \geq 1} \frac{(-1)^{n_{1}+\cdots+n_{r}}}{n_{1}^{s_{1}} \cdots n_{r}^{s_{r}}},
$$

where $n \geq_{2} m$ means " $n \geq m$ with equality only if $n$ is even". Define the symmetrization of this series by

$$
T^{\Sigma}\left(s_{1}, \ldots, s_{r}\right)=\sum_{\sigma \in \Sigma_{r}} T\left(s_{\sigma_{1}}, \ldots, s_{\sigma_{r}}\right),
$$

where $\Sigma_{r}$ is the symmetric group.

Theorem 1 The coefficients of the Hirzebruch L-polynomials are given by

$$
h_{j_{1}, \ldots, j_{r}}=\frac{(-1)^{r}}{\alpha_{1} ! \cdots \alpha_{k} !} \frac{2^{2 k}}{\pi^{2 k}} T^{\Sigma}\left(2 j_{1}, \ldots, 2 j_{r}\right),
$$

where $\alpha_{\ell}$ counts how many of $j_{1}, \ldots, j_{r}$ are equal to $\ell$.

It is well-known that $h_{k}$ is positive for all $k$. In [8, Appendix A], it is argued that $h_{i, j}$ is always negative and that $h_{i, j, k}$ is always positive (following an argument attributed to Galatius in the case of $h_{i, j}$ ), and it is asked whether it has been proved in general that $(-1)^{r-1} h_{j_{1}, \ldots, j_{r}}$ is positive. We have not been able to locate such a result in the literature, but we can prove it using our formula. It follows from the following result.

Theorem 2 For all real $s_{1}, \ldots, s_{r}>1$,

$$
T\left(s_{1}, \ldots, s_{r}\right)<0 .
$$

Corollary 3 The coefficient $h_{j_{1}, \ldots, j_{r}}$ in the Hirzebruch L-polynomial $\mathrm{L}_{k}$ is non-zero for every partition $\left(j_{1}, \ldots, j_{r}\right)$ of $k$. It is negative if $r$ is even and positive if $r$ is odd. 
It is remarked in [8] that a similar pattern has been observed in the multiplicative sequence of polynomials associated with the $\hat{A}$-genus. The polynomials in question are

$$
\begin{aligned}
& \hat{\mathrm{A}}_{1}=-\frac{1}{24} \mathrm{p}_{1}, \\
& \hat{\mathrm{A}}_{2}=\frac{1}{5760}\left(-4 \mathrm{p}_{2}+7 \mathrm{p}_{1}^{2}\right), \\
& \hat{\mathrm{A}}_{3}=\frac{1}{967680}\left(-16 \mathrm{p}_{3}+44 \mathrm{p}_{2} \mathrm{p}_{1}-31 \mathrm{p}_{1}^{3}\right),
\end{aligned}
$$

These can be treated similarly. Let us write

$$
\hat{\mathrm{A}}_{k}=\sum a_{j_{1}, \ldots, j_{r}} \mathrm{p}_{j_{1}} \cdots \mathrm{p}_{j_{r}}
$$

where the sum is over all partitions $\left(j_{1}, \ldots, j_{r}\right)$ of $k$. Consider the series

$$
S\left(s_{1}, \ldots, s_{r}\right)=\sum_{n_{1} \geq \cdots \geq n_{r} \geq 1} \frac{1}{n_{1}^{s_{1}} \cdots n_{r}^{s_{r}}},
$$

and its symmetrization

$$
S^{\Sigma}\left(s_{1}, \ldots, s_{r}\right)=\sum_{\sigma \in \Sigma_{r}} S\left(s_{\sigma_{1}}, \ldots, s_{\sigma_{r}}\right)
$$

Theorem 4 The coefficients of the Â-polynomials are given by

$$
a_{j_{1}, \ldots, j_{r}}=\frac{(-1)^{r}}{\alpha_{1} ! \cdots \alpha_{k} !} \frac{1}{(2 \pi)^{2 k}} S^{\Sigma}\left(2 j_{1}, \ldots, 2 j_{r}\right) \text {. }
$$

In particular, the coefficient $a_{j_{1}, \ldots, j_{r}}$ is negative if $r$ is odd and positive if $r$ is even.

\section{Proofs}

The first step in our proof is to establish a formula that expresses the coefficient $h_{j_{1}, \ldots, j_{r}}$ as a linear combination of products $h_{k_{1}} \cdots h_{k_{\ell}}$. This generalizes the formulas for $h_{i, j}$ and $h_{i, j, k}$ found in [8]. In the appendix of [2], recursive formulas for computing $h_{j_{1}, \ldots, j_{r}}$ in terms of products $h_{k_{1}} \cdots h_{k_{\ell}}$ are given. Here we give an explicit closed formula. The result holds for arbitrary multiplicative sequences of polynomials (see $[3, \S 1]$ ).

Theorem 5 Let $K_{0}, K_{1}, K_{2}, \ldots$ be a multiplicative sequence of polynomials with

$$
K_{k}=\sum \lambda_{j_{1}, \ldots, j_{r}} \mathrm{p}_{j_{1}} \cdots \mathrm{p}_{j_{r}}
$$


The coefficients satisfy the relation

$$
\lambda_{j_{1}, \ldots, j_{r}}=\frac{1}{\alpha_{1} ! \cdots \alpha_{k} !} \sum_{\mathcal{P}}(-1)^{r-\ell} c_{\mathcal{P}} \lambda_{k_{1}} \cdots \lambda_{k_{\ell}}
$$

where $\alpha_{i}$ counts how many of $j_{1}, \ldots, j_{r}$ are equal to $i$, the sum is over all partitions $\mathcal{P}=\left\{P_{1}, \ldots, P_{\ell}\right\}$ of the set $\{1,2, \ldots, r\}$,

$$
c_{\mathcal{P}}=\left(\left|P_{1}\right|-1\right) ! \cdots\left(\left|P_{\ell}\right|-1\right) !
$$

and

$$
k_{m}=\sum_{i \in P_{m}} j_{i}
$$

Proof A multiplicative sequence of polynomials is determined by its characteristic power series

$$
Q(z)=\sum_{k=0}^{\infty} b_{k} z^{k}
$$

where $b_{k}=\lambda_{1, \ldots, 1}$ is the coefficient of $\mathrm{p}_{1}^{k}$ in $K_{k}$. Indeed, if we, as in [3], formally interpret the coefficients $b_{k}$ as elementary symmetric functions in $\beta_{1}^{\prime}, \ldots, \beta_{m}^{\prime}(m \geq k)$, so that

$$
1+b_{1} z+b_{2} z^{2}+\cdots+b_{m} z^{m}=\left(1+\beta_{1}^{\prime} z\right) \cdots\left(1+\beta_{m}^{\prime} z\right)
$$

then the coefficient $\lambda_{j_{1}, \ldots, j_{r}}$ is the monomial symmetric function in $\beta_{1}^{\prime}, \ldots, \beta_{m}^{\prime}$ (see [3, Lemma 1.4.1]).

Note that $\lambda_{k}$ equals the power sum $\sum_{i}\left(\beta_{i}^{\prime}\right)^{k}$. The product $\lambda_{k_{1}} \cdots \lambda_{k_{\ell}}$ is then the power sum symmetric function evaluated at $\beta_{i}^{\prime}$, and the claim follows from a general formula that expresses the monomial symmetric functions in terms of power sum symmetric functions, see Theorem 8 below.

The characteristic series of the Hirzebruch $L$-polynomials is

$$
\frac{\sqrt{z}}{\tanh \sqrt{z}}=1+\sum_{k=1}^{\infty} b_{k} z^{k}
$$

where

$$
b_{k}=(-1)^{k-1} \frac{2^{2 k}}{(2 k) !} B_{k}
$$


and $B_{k}$ are the Bernoulli numbers,

$$
B_{1}=\frac{1}{6}, B_{2}=\frac{1}{30}, B_{3}=\frac{1}{42}, \ldots,
$$

see $[3, \S 1.5]$.

As is well-known, the leading coefficient $h_{k}$ of $\mathrm{p}_{k}$ in $L_{k}$ is given by

$$
h_{k}=\frac{2^{2 k}\left(2^{2 k-1}-1\right)}{(2 k) !} B_{k},
$$

see [3, p.12]. In [8], the formula

$$
h_{k}=\zeta(2 k) \frac{2^{2 k}-2}{\pi^{2 k}},
$$

involving the Riemann zeta function,

$$
\zeta(s)=\sum_{n=1}^{\infty} \frac{1}{n^{s}}
$$

is used to argue that $h_{i, j}<0$ and $h_{i, j, k}>0$. From this point on, our argument will depart from that of [8]. A key observation is that we can express $h_{k}$ in terms of the alternating zeta function,

$$
\zeta^{*}(s)=\sum_{n=1}^{\infty} \frac{(-1)^{n-1}}{n^{s}}
$$

instead of the Riemann zeta function. It is well-known, and easily seen, that

$$
\zeta^{*}(s)=\left(1-2^{1-s}\right) \zeta(s)
$$

Moreover, the following holds for all positive integers $k$,

$$
\zeta^{*}(2 k)=\frac{\pi^{2 k}\left(2^{2 k-1}-1\right)}{(2 k) !} B_{k}
$$

Combining this with (2), we see that

$$
h_{k}=\frac{2^{2 k}}{\pi^{2 k}} \zeta^{*}(2 k) .
$$

From (1) we get

$$
h_{j_{1}, \ldots, j_{r}}=\frac{1}{\alpha_{1} ! \cdots \alpha_{k} !} \frac{2^{2 k}}{\pi^{2 k}} \sum_{\mathcal{P}}(-1)^{r-\ell} c_{\mathcal{P}} \zeta^{*}\left(2 k_{1}\right) \cdots \zeta^{*}\left(2 k_{\ell}\right),
$$

where the notation is as in Theorem 5. 
The next observation is that the sum in the right hand side bears a striking resemblance with the right hand side of Hoffman's formula [4, Theorem 2.2] (proved anew in Theorem 7 below), which relates multiple zeta values and products of zeta valuesthe only difference is that $\zeta^{*}$ appears instead of $\zeta$. The second step in the proof is then to find a Hoffman-like formula for $\zeta^{*}$. Here is what we were led to write down: Define, for real numbers $s_{1}, \ldots, s_{r}>1$,

$$
T\left(s_{1}, \ldots, s_{r}\right)=\sum_{n_{1} \geq 2 \cdots \geq 2} \frac{(-1)^{n_{1}+\cdots+n_{r}}}{n_{1}^{s_{1}} \cdots n_{r}^{s_{r}}}
$$

where $n \geq 2 m$ means " $n \geq m$ with equality only if $n$ is even". Then symmetrize, and define

$$
T^{\Sigma}\left(s_{1}, \ldots, s_{r}\right)=\sum_{\sigma \in \Sigma_{r}} T\left(s_{\sigma_{1}}, \ldots, s_{\sigma_{r}}\right)
$$

Here is our Hoffman-like formula. Together with (5) it implies Theorem 1.

Theorem 6 The following equality holds for all real $s_{1}, \ldots, s_{r}>1$,

$$
\sum_{\mathcal{P}}(-1)^{r-\ell} c_{\mathcal{P}} \zeta^{*}(\underline{s}, \mathcal{P})=(-1)^{r} T^{\Sigma}\left(s_{1}, \ldots, s_{r}\right)
$$

where the sum is over all partitions $\mathcal{P}=\left\{P_{1}, \ldots, P_{\ell}\right\}$ of $\{1,2, \ldots, r\}$ and

$$
c_{\mathcal{P}}=\left(\left|P_{1}\right|-1\right) ! \cdots\left(\left|P_{\ell}\right|-1\right) !, \quad \zeta^{*}(\underline{s}, \mathcal{P})=\zeta^{*}\left(\sum_{i \in P_{1}} s_{i}\right) \cdots \zeta^{*}\left(\sum_{i \in P_{\ell}} s_{i}\right)
$$

Proof This will follow by specialization of Theorem 10 below.

Next we turn to the proof of Theorem 2, which says that

$$
T\left(s_{1}, \ldots, s_{r}\right)<0
$$

for all real $s_{1}, \ldots, s_{r}>1$.

Proof of Theorem 2 The proof is in principle not more difficult than the proof that $\zeta^{*}(s)$ is positive; to see this one simply arranges the sum as

$$
\zeta^{*}(s)=\left(1-\frac{1}{2^{s}}\right)+\left(\frac{1}{3^{s}}-\frac{1}{4^{s}}\right)+\cdots+\left(\frac{1}{(2 k-1)^{s}}-\frac{1}{(2 k)^{s}}\right)+\cdots,
$$

and notes that the summands are positive. Since the series is absolutely convergent, we are free to rearrange as we please, as the reader will recall from elementary analysis. 
Towards the general case, introduce for $k \geq 1$ the auxiliary series

$$
T_{2 k}\left(s_{1}, \ldots, s_{r}\right)=\sum_{n_{1} \geq 2 \cdots \geq 2 n_{r} \geq 2 k} \frac{(-1)^{n_{1}+\cdots+n_{r}}}{n_{1}^{s_{1}} \cdots n_{r}^{s_{r}}} .
$$

Then one can argue using the following two equalities, whose verification we leave to the reader:

$$
\begin{aligned}
& T\left(s_{1}, \ldots, s_{r}\right)=\sum_{k \geq 1}\left(-\frac{1}{(2 k-1)^{s_{r}}}+\frac{1}{(2 k)^{s_{r}}}\right) T_{2 k}\left(s_{1}, \ldots, s_{r-1}\right), \\
& T_{2 k}\left(s_{1}, \ldots, s_{r}\right) \\
& \quad=\sum_{\ell \geq k} \sum_{j=1}^{r} \frac{1}{(2 \ell)^{s_{r}} \ldots(2 \ell)^{s_{j+1}}}\left(\frac{1}{(2 \ell)^{s_{j}}}-\frac{1}{(2 \ell+1)^{s_{j}}}\right) T_{2 \ell+2}\left(s_{1}, \ldots, s_{j-1}\right) .
\end{aligned}
$$

Here $T_{2 \ell+2}\left(s_{1}, \ldots, s_{j-1}\right)$ should be interpreted as 1 for $j=1$. The second equality may be used to show that $T_{2 k}\left(s_{1}, \ldots, s_{r}\right)$ is positive by induction on $r$. The first equality then shows that $T\left(s_{1}, \ldots, s_{r}\right)$ is negative.

Finally, we turn to the proof of Theorem 4. The argument turns out to be easier in this case. Recall that the $\hat{A}$-genus has characteristic series

$$
Q(z)=\frac{\sqrt{z} / 2}{\sinh (\sqrt{z} / 2)} .
$$

Let us write

$$
\hat{\mathrm{A}}_{k}=\sum a_{j_{1}, \ldots, j_{r}} \mathrm{p}_{j_{1}} \cdots \mathrm{p}_{j_{r}}
$$

where the sum is over all partitions $\left(j_{1}, \ldots, j_{r}\right)$ of $k$. By using the Cauchy formula (see [3, p.11]) one can calculate the coefficient $a_{k}$ of $\mathrm{p}_{k}$ in $\hat{\mathrm{A}}_{k}$. The result is

$$
a_{k}=\frac{(-1)^{k}}{2(2 k) !} B_{k}=-\frac{1}{(2 \pi)^{2 k}} \zeta(2 k) \text {. }
$$

It follows that

$$
a_{k_{1}} \cdots a_{k_{\ell}}=\frac{(-1)^{\ell}}{(2 \pi)^{2 k}} \zeta\left(2 k_{1}\right) \cdots \zeta\left(2 k_{\ell}\right),
$$

for every partition $\left(k_{1}, \ldots, k_{\ell}\right)$ of $k$. Theorem 5 then yields

$$
\begin{aligned}
a_{j_{1}, \ldots, j_{r}} & =\frac{1}{\alpha_{1} ! \cdots \alpha_{k} !} \sum_{\mathcal{P}}(-1)^{r-\ell} c_{\mathcal{P}} a_{k_{1}} \cdots a_{k_{\ell}} \\
& =\frac{1}{\alpha_{1} ! \cdots \alpha_{k} !} \frac{(-1)^{r}}{(2 \pi)^{2 k}} \sum_{\mathcal{P}} c_{\mathcal{P}} \zeta\left(2 k_{1}\right) \cdots \zeta\left(2 k_{\ell}\right) .
\end{aligned}
$$


The terms in the sum are clearly positive, so we see already from this expression that $(-1)^{r} a_{j_{1}, \ldots, j_{r}}>0$ for all partitions $\left(j_{1}, \ldots, j_{r}\right)$ of $k$. However, more can be said; the sum in the right hand side now not only resembles but is equal to the right hand side of another formula of Hoffman [4, Theorem 2.1]. In our notation this formula says that

$$
S^{\Sigma}\left(s_{1}, \ldots, s_{r}\right)=\sum_{\mathcal{P}} c_{\mathcal{P}} \zeta(\underline{s}, \mathcal{P}) .
$$

This proves Theorem 4.

\section{Combinatorics of infinite sums}

The proofs of Theorems 5 and 6, as well as of Hoffman's formula, share the same combinatorial underpinnings; this is the topic of the present section.

Recall that a partition of a set $S$ is a set of non-empty disjoint subsets,

$$
\pi=\left\{\pi_{1}, \ldots, \pi_{r}\right\}
$$

such that $S=\pi_{1} \cup \cdots \cup \pi_{r}$. Write $\ell(\pi)=r$ for the length of $\pi$. The set of partitions $\Pi_{S}$ is partially ordered by refinement, $\pi=\left\{\pi_{1} \ldots, \pi_{r}\right\} \leq \rho=\left\{\rho_{1}, \ldots, \rho_{\ell}\right\}$ if and only if there is a partition $\mathcal{P}=\left\{P_{1}, \ldots, P_{\ell}\right\}$ of the set $\{1,2, \ldots, r\}$ such that

$$
\rho_{i}=\bigcup_{j \in P_{i}} \pi_{j}, \quad 1 \leq i \leq \ell .
$$

We will write $\rho=\mathcal{P}(\pi)$ if (7) holds. Note that for every $\rho \geq \pi$ there is a unique partition $\mathcal{P}$ such that $\rho=\mathcal{P}(\pi)$.

We will consider certain formal power series in indeterminates $a_{n}$ for $a \in S$ and positive integers $n$. For a subset $T \subseteq S$, write

$$
f_{T}(n)=\prod_{a \in T} a_{n}
$$

For a partition $\pi=\left\{\pi_{1}, \ldots, \pi_{r}\right\}$ of $S$, consider the formal power series

$$
\begin{aligned}
& p_{\pi}=\sum_{n_{1}, \ldots, n_{r}} f_{\pi_{1}}\left(n_{1}\right) \cdots f_{\pi_{r}}\left(n_{r}\right), \\
& m_{\pi}=\sum_{\substack{n_{1}, \ldots, n_{r} \\
\text { distinct }}} f_{\pi_{1}}\left(n_{1}\right) \cdots f_{\pi_{r}}\left(n_{r}\right) .
\end{aligned}
$$

It is then immediate that

$$
p_{\pi}=\sum_{\rho \geq \pi} m_{\rho} .
$$


By applying the Möbius inversion formula (see e.g. [6, Proposition 3.7.2]), we get

$$
m_{\pi}=\sum_{\rho \geq \pi} \mu(\pi, \rho) p_{\rho} .
$$

The Möbius function of $\Pi_{S}$ is given by

$$
\mu(\pi, \rho)=(-1)^{\ell(\pi)-\ell(\rho)}\left(b_{1}-1\right) ! \ldots\left(b_{\ell(\rho)}-1\right) !,
$$

where the number

$$
b_{i}=b_{i}(\pi, \rho)=\left|P_{i}\right|
$$

counts how many ' $\pi$-blocks' $\rho_{i}$ consists of, see e.g. [6, Example 3.10.4].

Let us first note that this gives a neat proof of Hoffman's formula (though we would be surprised if this has not been noticed before). Recall that the multiple zeta function is defined by

$$
\zeta\left(s_{1}, \ldots, s_{r}\right)=\sum_{n_{1}>\cdots>n_{r} \geq 1} \frac{1}{n_{1}^{s_{1}} \cdots n_{r}^{s_{r}}}
$$

for real $s_{1}, \ldots, s_{r}>1$.

Theorem 7 (Hoffman [4, Theorem 2.2])

$$
\sum_{\sigma \in \Sigma_{r}} \zeta\left(s_{\sigma_{1}}, \ldots, s_{\sigma_{r}}\right)=\sum_{\mathcal{P}}(-1)^{r-\ell} c_{\mathcal{P}} \zeta(\underline{s}, \mathcal{P})
$$

where the sum is over all partitions $\mathcal{P}=\left\{P_{1}, \ldots, P_{\ell}\right\}$ of $\{1,2, \ldots, r\}$ and

$$
c_{\mathcal{P}}=\left(\left|P_{1}\right|-1\right) ! \cdots\left(\left|P_{\ell}\right|-1\right) !, \quad \zeta(\underline{s}, \mathcal{P})=\zeta\left(\sum_{i \in P_{1}} s_{i}\right) \cdots \zeta\left(\sum_{i \in P_{\ell}} s_{i}\right) .
$$

Proof Take $S=\{1,2, \ldots, r\}$ and substitute $a_{n}$ by $\frac{1}{n^{s a}}$ for $a \in S$ in (8).

Secondly, we will use (8) to express the monomial symmetric functions in terms of power sum symmetric functions. We refer to [7, Chapter 7] for a pleasant introduction to symmetric functions. For an integer partition $I=\left(i_{1}, \ldots, i_{r}\right) \vdash k$, recall that the power sum symmetric function $p_{I}$ is the formal power series in indeterminates $x_{1}, x_{2}, \ldots$ defined by $p_{I}=p_{i_{1}} \cdots p_{i_{r}}$, where

$$
p_{j}=\sum_{i} x_{i}^{j}
$$

The monomial symmetric function $m_{I}$ is defined as the sum of all pairwise distinct monomials of the form $x_{\sigma_{1}}^{i_{1}} \cdots x_{\sigma_{r}}^{i_{r}}$. 
Theorem 8 For every $k \geq 1$ and every integer partition $I=\left(i_{1}, \ldots, i_{r}\right)$ of $k$,

$$
m_{I}=\frac{1}{\alpha_{1} ! \cdots \alpha_{k} !} \sum_{\mathcal{P}}(-1)^{r-\ell} c_{\mathcal{P}} p_{J}
$$

where the sum is over all partitions $\mathcal{P}=\left\{P_{1}, \ldots, P_{\ell}\right\}$ of the set $\{1,2, \ldots, r\}$, the number $\alpha_{j}$ counts how many of $i_{1}, \ldots, i_{r}$ are equal to $j$, and $J=\left(j_{1}, \ldots, j_{\ell}\right)$ is given by

$$
j_{u}=\sum_{v \in P_{u}} i_{v}, \quad 1 \leq u \leq \ell
$$

Proof Let $S$ be any set with $k$ elements. Perform the substitution $a_{n}=x_{n}$ for each $a \in S$ in the equality (8) and note that this takes $p_{\pi}$ to $p_{I}$ and $m_{\pi}$ to $\alpha_{1} ! \cdots \alpha_{k} ! m_{I}$, where $I=\left(\left|\pi_{1}\right|, \ldots,\left|\pi_{r}\right|\right)$ is the integer partition underlying the set partition $\pi$ (assuming, as we may, $\left|\pi_{1}\right| \geq \cdots \geq\left|\pi_{r}\right|$ ).

Next, we turn to the result that will specialize to our formula in Theorem 6. Consider the following alternating version of $p_{\pi}$ :

$$
\bar{p}_{\pi}=\sum_{n_{1}, \ldots, n_{r}}(-1)^{n_{1}+\cdots+n_{r}} f_{\pi_{1}}\left(n_{1}\right) \cdots f_{\pi_{r}}\left(n_{r}\right) .
$$

For an ordered partition $\tilde{\pi}=\left(\pi_{1}, \ldots, \pi_{r}\right)$ we define

$$
T_{\widetilde{\pi}}=\sum_{n_{1} \geq 2 \cdots \geq 2 n_{r}}(-1)^{n_{1}+\cdots+n_{r}} f_{\pi_{1}}\left(n_{1}\right) \cdots f_{\pi_{r}}\left(n_{r}\right) .
$$

Then for an unordered partition $\pi=\left\{\pi_{1}, \ldots, \pi_{r}\right\}$, define

$$
T_{\pi}^{\Sigma}=\sum_{\tilde{\pi}} T_{\tilde{\pi}}
$$

where the sum is over the $r$ ! ordered partitions $\tilde{\pi}$ whose underlying unordered partition is $\pi$.

Lemma 9 For every partition $\pi \in \Pi_{S}$,

$$
\sum_{\rho \geq \pi}(-1)^{\ell(\rho)} \ell(\rho) !=(-1)^{\ell(\pi)} .
$$

Proof We may without loss of generality assume that $\pi$ is the minimal element, because the poset $\left\{\rho \in \Pi_{S} \mid \rho \geq \pi\right\}$ is isomorphic to the poset $\Pi_{\pi}$ of partitions of the set $\pi$. Then $n=\ell(\pi)$ is the number of elements of $S$. The number of partitions of 
length $k$ in $\Pi_{S}$ is equal to the Stirling number of the second kind $S(n, k)$, see e.g. [6, Example 3.10.4]. Thus,

$$
\sum_{\rho \in \Pi_{S}}(-1)^{\ell(\rho)} \ell(\rho) !=\sum_{k=1}^{n}(-1)^{k} S(n, k) k ! .
$$

By plugging in $x=-1$ in the well-known identity

$$
\sum_{k=1}^{n} S(n, k)(x)_{k}=x^{n}
$$

where $(x)_{k}=x(x-1)(x-2) \cdots(x-k+1)$, we see that (9) equals $(-1)^{n}$.

Theorem 10 For every partition $\pi$,

$$
(-1)^{\ell(\pi)} T_{\pi}^{\Sigma}=\sum_{\rho \geq \pi}(-1)^{\ell(\rho)} \mu(\pi, \rho) \bar{p}_{\rho} .
$$

Proof By Möbius inversion, the equality is equivalent to

$$
(-1)^{\ell(\pi)} \bar{p}_{\pi}=\sum_{\rho \geq \pi}(-1)^{\ell(\rho)} T_{\rho}^{\Sigma},
$$

and we proceed to prove (11). It is clear that both sides can be written as linear combinations of series of the form

$$
m_{v, e}=\sum_{\substack{n_{1}, \ldots, n_{m} \text { distinct } \\ n_{i} \equiv 2 e_{i}}} f_{v_{1}}\left(n_{1}\right) \cdots f_{v_{m}}\left(n_{m}\right),
$$

for various $v=\left\{v_{1}, \ldots, v_{m}\right\} \geq \pi$, where $e$ is an assignment of a parity $e_{i} \in\{0,1\}$ to each $v_{i}$. For example, if $v=\{\{a, b\},\{c\}\}$ and $e$ assigns 1 to $\{a, b\}$ and 0 to $\{c\}$, then

$$
m_{v, e}=\sum_{n_{1} \text { odd, } n_{2} \text { even }} a_{n_{1}} b_{n_{1}} c_{n_{2}} .
$$

The question is with what coefficients $m_{v, e}$ will appear in the respective sides of (11). For the left hand side this is not difficult: $m_{v, e}$ appears in $(-1)^{\ell(\pi)} \bar{p}_{\pi}$ with coefficient

$$
\operatorname{sgn}(\pi, v, e)=(-1)^{v_{1}\left(e_{1}-1\right)+\cdots+v_{m}\left(e_{m}-1\right)},
$$

where $v_{i}$ is the number of $\pi$-blocks in $v_{i}$.

The right hand side requires a little more effort-and notation. It is clear that $m_{v, e}$ appears in $T_{\rho}^{\Sigma}$ only if $\nu \geq \rho$ and $e$ assigns an even value to $v_{i}$ whenever $v_{i}$ consists of more than one $\rho$-block. This can be reformulated as saying that $v \geq \rho \geq e(v)$, where $e(v) \leq v$ is the partition that keeps $v_{i}$ intact if $e_{i}$ is odd and splits $v_{i}$ completely if $e_{i}$ is 
even. Or more precisely, $e(v)$ is the smallest element below $v$ that contains $v_{i}$ whenever $e_{i}$ is odd. Since we symmetrize, there will be repetitions; for $v \geq \rho \geq e(v)$, the term involving $m_{v, e}$ will be repeated $b_{\rho, v}=b_{1} ! \cdots b_{m}$ ! times in $T_{\rho}^{\Sigma}$, where $b_{i}$ is the number of $\rho$-blocks in $v_{i}$. Thus, the coefficient of $m_{v, e}$ in $(-1)^{\ell(\rho)} T_{\rho}^{\Sigma}$ is $\operatorname{sgn}(\rho, v, e) b_{\rho, v}$. It follows that the coefficient of $m_{v, e}$ in $\sum_{\rho \geq \pi}(-1)^{\ell(\rho)} T_{\rho}^{\Sigma}$ is

$$
\sum_{\nu \geq \rho \geq e(v) \vee \pi} \operatorname{sgn}(\rho, v, e) b_{\rho, v}=\sum_{\nu \geq \rho \geq e(v) \vee \pi}(-1)^{b_{1}\left(e_{1}-1\right)+\cdots+b_{m}\left(e_{m}-1\right)} b_{1} ! \cdots b_{m} !
$$

where $e(\nu) \vee \pi$ is the least upper bound of $e(\nu)$ and $\pi$.

Put $\pi_{(i)}=\left\{\pi_{j} \in \pi: \pi_{j} \subseteq v_{i}\right\}$ and $\nu_{(i)}=\left\{v_{i}\right\}$. We then have an isomorphism of posets $[e(v) \vee \pi, v] \cong \prod_{i: e_{i}=0}\left[\pi_{(i)}, v_{(i)}\right]$. Under this isomorphism $\rho \in[e(\nu) \vee \pi, \nu]$ is sent to $\rho_{(i)}=\left\{\rho_{j} \in \rho: \rho_{j} \subseteq v_{i}\right\} \in\left[\pi_{(i)}, v_{(i)}\right]$. Note that $b_{i}$ is the length of $\rho_{(i)}$. We now find that the sum (13) factors as a product

$$
\prod_{i: e_{i}=0} \sum_{\nu_{(i)} \geq \rho_{(i)} \geq \pi_{(i)}}(-1)^{b_{i}} b_{i} !
$$

By Lemma 9, this is equal to

$$
\prod_{i: e_{i}=0}(-1)^{v_{i}}
$$

This shows that (13) equals (12), and the theorem is proved.

To prove Theorem 6 , take $S=\{1,2, \ldots, r\}$ and substitute $a_{n}$ by $\frac{1}{n^{s a}}$ in (10).

Acknowledgements We thank Don Zagier and Matthias Kreck for valuable comments. The impetus for this work was a question from Oscar Randal-Williams to the first author about certain points in [1]. The first author was supported by the Swedish Research Council through Grant No. 2015-03991.

Open Access This article is distributed under the terms of the Creative Commons Attribution 4.0 International License (http://creativecommons.org/licenses/by/4.0/), which permits unrestricted use, distribution, and reproduction in any medium, provided you give appropriate credit to the original author(s) and the source, provide a link to the Creative Commons license, and indicate if changes were made.

\section{References}

1. Berglund, A., Madsen, I.: Rational homotopy theory of automorphisms of manifolds. arXiv:1401.4096v2 [math.AT]

2. Fowler, J., Su, Z.: Smooth manifolds with prescribed rational cohomology ring. Geom. Dedicata 182, 215-232 (2016)

3. Hirzebruch, F.: Topological Methods in Algebraic Geometry, 3rd edn. Springer, Berlin (1966). (Die Grundlehren der Mathematischen Wissenschaften, Band 131)

4. Hoffman, M.E.: Multiple harmonic series. Pac. J. Math. 152(2), 275-290 (1992)

5. Milnor, J.W., Stasheff, J.D.: Characteristic Classes. Annals of Mathematics Studies, vol. 76. Princeton University Press, Princeton (1974)

6. Stanley, R.P.: Enumerative Combinatorics, vol. 1. Cambridge University Press, Cambridge (1997). Cambridge Studies in Advanced Mathematics, 49 
7. Stanley, R.P.: Enumerative Combinatorics, vol. 2. Cambridge University Press, Cambridge (1999). Cambridge Studies in Advanced Mathematics, 62

8. Weiss, M.S.: Dalian notes on rational Pontryagin classes. arXiv:1507.00153v3 [math.AT] 